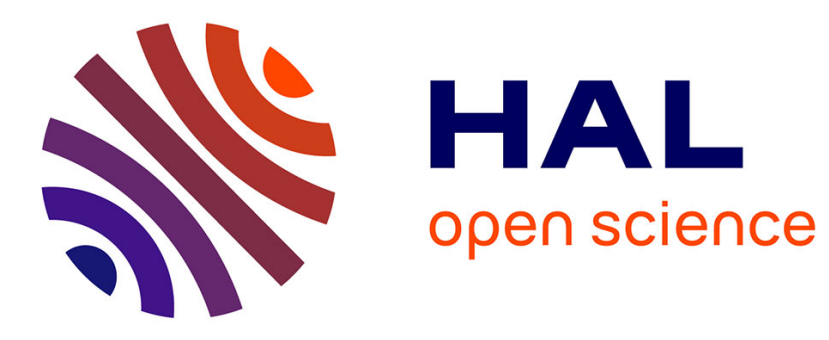

\title{
Robust control of pneumatic actuators based on a simplified model with delayed input
}

E Edjekouane, Samer Riachy, M Ghanes, Jean-Pierre Barbot

\section{To cite this version:}

E Edjekouane, Samer Riachy, M Ghanes, Jean-Pierre Barbot. Robust control of pneumatic actuators based on a simplified model with delayed input. 13th International Workshop on Variable Structure Systems, Jun 2014, Nante, France. pp.1 - 6, 10.1109/VSS.2014.6881117 . hal-01084398

\section{HAL Id: hal-01084398 \\ https://inria.hal.science/hal-01084398}

Submitted on 19 Nov 2014

HAL is a multi-disciplinary open access archive for the deposit and dissemination of scientific research documents, whether they are published or not. The documents may come from teaching and research institutions in France or abroad, or from public or private research centers.
L'archive ouverte pluridisciplinaire HAL, est destinée au dépôt et à la diffusion de documents scientifiques de niveau recherche, publiés ou non, émanant des établissements d'enseignement et de recherche français ou étrangers, des laboratoires publics ou privés. 


\title{
Robust Control of Pneumatic Actuators Based on a Simplified Model With Delayed Input
}

\author{
E. Edjekouane, S. Riachy, M. Ghanes and J-P. Barbot
}

\begin{abstract}
Due to frictions and air compressibility, the dynamics of pneumatic actuators is often described by a complex fourth-order non linear model. Therefore, simplifying the model of pneumatic actuators is of prime interest to design a controller. In this paper a simple second order model is proposed by modeling the pressure dynamics with a pure time delay on the control input. The Artstein transformation is applied to this model to get a delay-free second order system. Then the delayfree system is stabilized using a robust nonlinear controller. The relevance of the proposed approach is demonstrated through experimental tests.
\end{abstract}

\section{INTRODUCTION}

The control of pneumatic actuators remains a challenge till today in order to obtain better results in positioning, tracking and in terms of robustness (disturbance rejection). This challenge is raised by both industrial and academic partners, across several applications and publications. The pneumatic actuators have a wide field of use ranging from simple process to the complex ones, like in production line, aeronautic and automotive industry. This large place they hold is due to their ease of maintenance, rigidity and safety. However, their dynamic modeling is quite difficult since, the description of the air dynamic is often based on empirical considerations. Moreover, accurate positioning of such an actuator is a difficult task due to the presence of discontinuous friction and air compressibility.

The model used to design controllers for this kind of actuators is often a fourth order model (see [4], [7], [8], [9], [10]). The major drawback of such a model is the difficulty of controller design and closed loop stability analysis. The first contribution of the present work is the introduction of a simplified model which is based on the following observation. A pure time delay is used to model the pressures dynamics. This delay occurs between the moment of the opening of the servovalve and the moment when the force acts on the piston. Consequently, a double integrator with delayed input is obtained. This idea has been raised in the conclusion of [9].

The observation that the pressure dynamics induces a pure time delay was already invoked in [10]. In order to minimize the influence of the delay, the adopted method consisted in fully opening the servovalve during a very short time at the beginning of a piston displacement in order to force quick pressure establishment in the cylinder compartment.

E. Edjekouane, S. Riachy, M. Ghanes and J-P Barbot are with ECSLab/ENSEA, France. e-mail: essaid.edjekouane@ensea.fr, riachy@ensea.fr, ghanes@ensea.fr, barbot@ensea.fr

S. Riachy and J-P. Barbot are also with Non-A team, INRIA Lille-NordEurope.
The second contribution of this paper is the design of a robust controller based on the proposed second order model. The control synthesis is performed in two steps. First, the Artstein transformation is applied to the second order model in order to obtain a system free of delay. Then, a controller based on the concepts of homogeneity and finite-time stability [2] is used to stabilize the transformed system. The proposed controller can be seen as a continuous approximation of a standard second order sliding mode. The stability of the closed loop is analyzed with and without perturbations.

It is clear from [10], that the delay takes different values depending on the opening rate of the servovalve. As a matter of fact, the bigger the opening rate is, the faster the pressure builds up in the cylinder chamber. The delay decreases then as the opening rate of the servovalve increases. However, a constant delay is assumed. The validity of this assumption is supported by the experimental system positioning accuracy.

The paper is organized as follows. Section I recalls the fourth order model, introduces the second order model with input delay and experimentally estimates the delay value. Section $\Pi$ iI is dedicated to the control design while section IV]deals with the stability analysis of the closed loop system. The discretization of the controller is detailed in section V Section VI presents the experimental tests.

\section{DYNAMIC MODEL}

\section{A. The fourth order model}

The derivation of the dynamic equations of the pneumatic actuator can be found in [9], [10] and [11]. It comes to a model where the state vector is of dimension 4 . With $y$ to denote the position of the piston, $v$ its velocity, $P_{1}$ and $P_{2}$ the pressures in both compartments of the cylinder, the dynamic equations writes:

$$
\begin{aligned}
\dot{y} & =v \\
\dot{v} & =M^{-1}\left(P_{1} A_{1}-P_{2} A_{2}+\Delta\right) \\
\dot{P}_{1} & =-\frac{k P_{1} v}{L+y}+\frac{\Omega}{A_{1}}\left\{\frac{1+\sigma(u)}{2} \frac{\gamma_{1 b} P_{s}}{L+y}-\frac{1-\sigma(u)}{2} \frac{\gamma_{1 e} P_{1}}{L+y}\right\}|u| \\
\dot{P}_{2} & =\frac{k P_{2} v}{L-y}+\frac{\Omega}{A_{2}}\left\{\frac{1-\sigma(u)}{2} \frac{\gamma_{2 b} P_{s}}{L-y}-\frac{1+\sigma(u)}{2} \frac{\gamma_{2 e} P_{2}}{L-y}\right\}|u|,
\end{aligned}
$$

where $\Omega=k \sqrt{R k T} A_{o} / U, \sigma(u)$ denotes the signum function 1 and $A_{o}$ the area of the orifice of the servovalve. The control $u$ represents the input voltage to the servovalve while $U$ denotes the maximal input voltage $(|u| \leq U) . P_{s}$ denotes

$$
{ }^{1} \sigma(u)=1 \text { if } u>0, \sigma(u)=0 \text { if } u=0, \sigma(u)=-1 \text { if } u<0 .
$$


the supply pressure. $\Delta$ represents an external load and can also represent gravity when the cylinder is mounted in a vertical position. $T$ denotes the air temperature, assumed to be constant. The heat coefficient for air is $k$, while $R$ is the perfect gas constant. $2 L$ denotes the total length of the cylinder while $M$ the mass of the moving part (piston, payload etc.). $\gamma_{1 b}, \gamma_{2 e}, \gamma_{1 e}$ and $\gamma_{2 b}$ are given by:

$$
\begin{aligned}
& \gamma_{1 b}=\left\{\begin{array}{ccc}
\sqrt{\frac{2}{k-1}}\left(\frac{P_{1}}{P_{s}}\right)^{\frac{k+1}{2 k}} \sqrt{\left(\frac{P_{1}}{P_{s}}\right)^{\frac{1-k}{k}}-1} & \text { if } & \frac{P_{1}}{P_{s}} \geq 0.528 \\
0.58 & \text { if } & \frac{P_{1}}{P_{s}}<0.528 .
\end{array}\right. \\
& \gamma_{2 e}=\left\{\begin{array}{ccc}
\sqrt{\frac{2}{k-1}}\left(\frac{P_{a}}{P_{2}}\right)^{\frac{k+1}{2 k}} \sqrt{\left(\frac{P_{a}}{P_{2}}\right)^{\frac{1-k}{k}}-1} & \text { if } & \frac{P_{a}}{P_{2}} \geq 0.528 \\
0.58 & \text { if } & \frac{P_{a}}{P_{2}}<0.528 .
\end{array}\right. \\
& \gamma_{1 e}=\left\{\begin{array}{ccc}
\sqrt{\frac{2}{k-1}}\left(\frac{P_{a}}{P_{1}}\right)^{\frac{k+1}{2 k}} \sqrt{\left(\frac{P_{a}}{P_{1}}\right)^{\frac{1-k}{k}}-1} & \text { if } & \frac{P_{a}}{P_{1}} \geq 0.528 \\
0.58 & \text { if } & \frac{P_{a}}{P_{1}}<0.528 .
\end{array}\right. \\
& \gamma_{2 b}=\left\{\begin{array}{ccc}
\sqrt{\frac{2}{k-1}}\left(\frac{P_{2}}{P_{s}}\right)^{\frac{k+1}{2 k}} \sqrt{\left(\frac{P_{2}}{P_{s}}\right)^{\frac{1-k}{k}}-1} & \text { if } & \frac{P_{2}}{P_{s}} \geq 0.528 \\
0.58 & \text { if } & \frac{P_{2}}{P_{s}}<0.528 .
\end{array}\right.
\end{aligned}
$$

and $P_{a}$ denotes atmospheric pressure.

Instead of this model, a second order model with an input delay is proposed. The validity of such a model for the control design purpose is supported by the experimental results. In addition, an interpretation via singular perturbation theory can be given [5]. In fact a two-time scale analysis can be conducted with the fast dynamics consisting of the air pressure and a slow one consisting of the mechanical piston.

\section{B. The proposed model with input delay}

The control design is based on the following second order model:

$$
\begin{aligned}
& \dot{x}_{1}=x_{2} \\
& \dot{x}_{2}=M G u(t-h)+p
\end{aligned}
$$

where $x_{1}$ and $x_{2}$ represent the piston position and velocity respectively. The delay is denoted by $h$ while $M$ denotes the piston and payload mass. Friction forces and gravity are represented by $p$ while $G$ represents an open loop gain. As previously discussed in section I, the delay $h$ is not necessarily constant. However, the following assumption is adopted.

Hypothesis 1: The delay $h>0$ is assumed to have a bounded constant value.

\section{Estimation of the delay}

We now proceed to an estimation of the delay $h$. This is done by open loop tests, where variable delay is observed ranging from 0.01 to 0.06 second as shown in figure 1 . In fact, the experiment confirm that the delay decreases as the opening rate of the servovalve increases. However, as required for the control design, the assumption of a constant delay is adopted. The validity of this assumption is confirmed by the experiments.

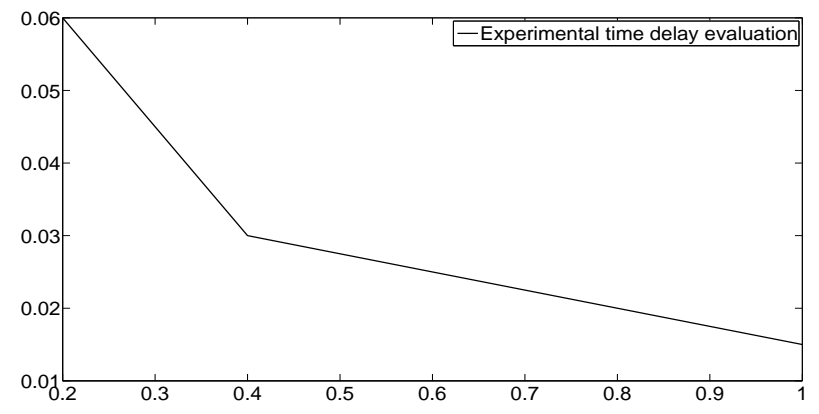

Fig. 1. Time delay (second) versus percentage of servovalve opening

\section{CONTROL DESIGN}

The Artstein transformation [1] permits to get rid of the delay. For the system (1), it is given by:

$$
\begin{aligned}
& z_{1}=x_{1}+h x_{2}+M G \int_{t-h}^{t}(t-\tau) u(\tau) d \tau \\
& z_{2}=x_{2}+M G \int_{t-h}^{t} u(\tau) d \tau .
\end{aligned}
$$

Differentiating (2) and using (1) lead to the delay-free system

$$
\begin{aligned}
& \dot{z}_{1}=z_{2}+h p \\
& \dot{z}_{2}=M G u+p .
\end{aligned}
$$

In the sequel, the following notation is used

$$
\lfloor\xi\rceil^{\alpha}=\operatorname{sign}(\xi)|\xi|^{\alpha}, \xi \in \mathbb{R}, \alpha>0
$$

where $|\cdot|$ denotes the Euclidean norm and corresponds to the absolute value for scalar entries. Note that the following rules for derivative are verified except at $\xi=0$, where the derivative is not defined: $\frac{d\lfloor\xi\rceil^{\alpha}}{d \xi}=\alpha|\xi|^{\alpha-1}$ and $\frac{d|\xi|^{\alpha}}{d \xi}=\alpha\left\lfloor\left.\xi\right|^{\alpha-1}, \forall \xi \epsilon\right.$ $\mathbb{R} \backslash\{0\}$. The control input

$$
u=-k_{1}\left\lfloor\dot{z}_{1}\right\rceil^{\alpha}-k_{2}\left\lfloor z_{1}+\frac{k_{3}}{2-\alpha}\left\lfloor\dot{z}_{1}\right\rceil^{2-\alpha}\right\rceil^{\frac{\alpha}{2-\alpha}}, \alpha \in[0,1],
$$

taken from [2] with positive constants $k_{1}, k_{2}$ and $k_{3}$, leads to the closed loop system:

$$
\begin{aligned}
& \dot{z}_{1}=z_{2}+h p \\
& \dot{z}_{2}=-K_{1}\left\lfloor z_{2}+h p\right\rceil^{\alpha}-K_{2}\left\lfloor z_{1}+K_{3}\left\lfloor z_{2}+h p\right\rceil^{2-\alpha}\right\rceil^{\frac{\alpha}{2-\alpha}}+p
\end{aligned}
$$

$K_{1}=M G k_{1}, K_{2}=M G k_{2}, K_{3}=\frac{k_{3}}{2-\alpha}$. In (4), $\dot{z}_{1}$ can be computed by using a differentiator. The control (4) with $\alpha \epsilon$ $[0,1)$ ensures, according to [3], a finite time stabilzation of (5) with $p=0$. A bloc diagram of the closed loop system is given in figure 2 .

In particular, with $\alpha=0$ the system (5) leads to:

$$
\begin{aligned}
& \dot{z}_{1}=z_{2}+h p \\
& \dot{z}_{2}=-K_{1} \operatorname{sign}\left(z_{2}+h p\right)-K_{2} \operatorname{sign}\left(z_{1}+K_{3}\left\lfloor z_{2}+h p\right\rceil^{2}\right)+p,
\end{aligned}
$$

which is a standard second order sliding mode system with unmatched perturbation. Note that due to the perturbation a chattering may appear near the equilibrium $z_{1}=z_{2}=0$. 


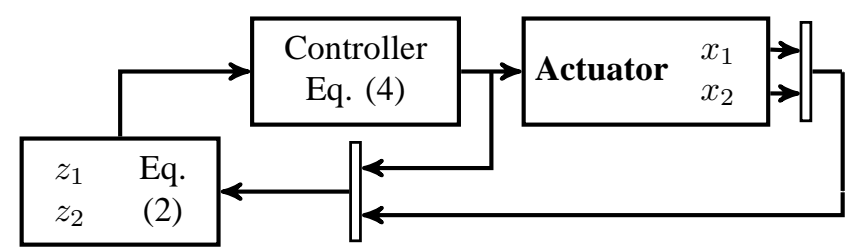

Fig. 2. Bloc diagram of the closed loop system

\section{STABILITY ANALYSIS}

The stability analysis is conducted in two steps. In section IV-A the unperturbed system $(p=0)$ is analyzed while the perturbed one is investigated in section IV-B

\section{A. Stability of the unperturbed system}

The system (5) with $p=0$ leads to,

$$
\begin{aligned}
& \dot{z}_{1}=z_{2} \\
& \dot{z}_{2}=-K_{1}\left\lfloor z_{2}\right\rceil^{\alpha}-K_{2}\left\lfloor z_{1}+K_{3}\left\lfloor z_{2}\right\rceil^{2-\alpha}\right\rceil^{\frac{\alpha}{2-\alpha}} .
\end{aligned}
$$

Proposition 4.1: The system (7) is asymptotically stable. Consequently, $x_{1}=x_{2}=0$ is an asymptotically stable equilibrium.

Proof: Consider the Lyapunov function

$$
V=K_{1} \frac{2-\alpha}{2}\left|z_{1}+\frac{k_{3}}{2-\alpha}\left\lfloor z_{2}\right\rceil^{2-\alpha}\right|^{\frac{2}{2-\alpha}}+\frac{\gamma}{2} z_{2}^{2}
$$

with, $\gamma$ a positive constant, chosen such that $K_{1}\left(1-K_{1} k_{3}\right)=$ $\gamma K_{2}$. The derivative of 8 is given by:

$$
\dot{V}=-K_{1} K_{2} k_{3}\left|z_{2}\right|^{1-\alpha} \mid z_{1}+\frac{k_{3}}{2-\alpha}\left\lfloor\left.\left. z_{2}\right|^{2-\alpha}\right|^{\frac{2 \alpha}{2-\alpha}}-\gamma K_{1}\left|z_{2}\right|^{1+\alpha}\right.
$$

which is negative semidefinite. Then by LaSalle's theorem, $\left(z_{1}, z_{2}\right)=(0,0)$ is an asymptotically stable equilibrium point and consequently is $\left(x_{1}, x_{2}\right)=(0,0)$ according to (2).

\section{B. Stability of the perturbed system}

Consider now, the perturbed system (5). With, $y_{1}=z_{1}$, $y_{2}=\dot{z}_{1}$ and $y=\left[\begin{array}{ll}y_{1} & y_{2}\end{array}\right]^{T}$, it can be rewritten as follows:

$$
\begin{aligned}
& \dot{y}_{1}=y_{2} \\
& \dot{y}_{2}=-K_{1}\left\lfloor y_{2}\right\rceil^{\alpha}-K_{2}\left\lfloor y_{1}+\frac{k_{3}}{2-\alpha}\left\lfloor y_{2}\right\rceil^{2-\alpha}\right\rceil^{\frac{\alpha}{2-\alpha}}+p .
\end{aligned}
$$

Hypothesis 2: The perturbation $p$ is nonzero but constant. Moreover, a positive constant $\bar{p}$ exists such that $|p|<\bar{p}$.

Proposition 4.2: Let assumptions 1 and 2 be $\quad$ verified. $\begin{aligned} & \text { Set } \theta_{1} \\ & \max \left(\frac{2 k_{3}}{2-\alpha}\left[\frac{\left(K_{1} k_{3}+\gamma\right) \bar{p}}{\gamma K_{1}}\right]^{\frac{2-\alpha}{1+\alpha}}, 2\left(\frac{2 \bar{p}}{K_{2}}\right)^{\frac{2-\alpha}{\alpha}}, 2\left[\frac{\left(1-K_{1} k_{3}\right) \bar{p}}{4 K_{1} K_{2} k_{3}}\right]^{\frac{2-\alpha}{\alpha}}\right)\end{aligned}$ and $\theta_{2}=\max \left(\frac{k_{3}}{2-\alpha}\left[\frac{\left(K_{1} k_{3}+\gamma\right) \bar{p}}{\gamma K_{1}}\right]^{\frac{2-\alpha}{1+\alpha}}, 2\left(\frac{2 \bar{p}}{K_{2}}\right)^{\frac{2-\alpha}{\alpha}}\right.$, $\left.2\left[\frac{\left(1-K_{1} k_{3}\right) \bar{p}}{4 K_{1} K_{2} k_{3}}\right]^{\frac{2-\alpha}{\alpha}}\right)$ with $\gamma=\frac{K_{1}\left(1-K_{1} k_{3}\right)}{K_{2}}$ and $\alpha \in(0,1)$. Assume that $\bar{p}$ is sufficiently small such that $\theta_{1}<1$ and $\theta_{2}<1$. Then the ball

$$
\mathbb{B}=\left\{\left(y_{1}, y_{2}\right) ;\left|y_{1}\right| \leq \theta_{1},\left|y_{2}\right| \leq \theta_{2}\right\}
$$

attracts any trajectory of (9) initialized within the compact set

$$
\begin{gathered}
\mathbb{B}_{0}=\left\{\left(y_{1}, y_{2}\right) ;\left|y_{1}\right| \leq 1,\left|y_{2}\right| \leq 1,\left|y_{1}\right|+\frac{k_{3}}{2-\alpha}\left|y_{2}\right|^{2-\alpha} \leq 1\right\} . \\
\text { Proof: Consider the Lyapunov function: }
\end{gathered}
$$

$$
V=K_{1} \frac{2-\alpha}{2}\left|y_{1}+\frac{k_{3}}{2-\alpha}\left\lfloor y_{2}\right\rceil^{2-\alpha}\right|^{\frac{2}{2-\alpha}}+\frac{\gamma}{2} y_{2}^{2}
$$

and take $\gamma$ in order to satisfy $K_{1}\left(1-K_{1} k_{3}\right)=\gamma K_{2}$.

The derivative of $V$ is rewritten as:

$$
\begin{aligned}
\dot{V} & \leq-K_{1} \mid y_{1}+\frac{k_{3}}{2-\alpha}\left\lfloor\left.\left. y_{2}\right|^{2-\alpha}\right|^{\frac{\alpha}{2-\alpha}} k_{3}\left|y_{2}\right|^{1-\alpha} \times\right. \\
& \left\{K_{2} \mid y_{1}+\frac{k_{3}}{2-\alpha}\left\lfloor\left.\left. y_{2}\right|^{2-\alpha}\right|^{\frac{\alpha}{2-\alpha}}-\bar{p}\right\}\right. \\
& -\gamma\left|y_{2}\right|\left[K_{1}\left|y_{2}\right|^{\alpha}-\bar{p}\right] .
\end{aligned}
$$

Set $a=\left|y_{1}+\frac{k_{3}}{2-\alpha}\left\lfloor y_{2}\right\rceil^{2-\alpha}\right|$ and consider the following cases.

- Pick a positive constant $\theta_{1}$ and assume that $\left|y_{1}\right|>\theta_{1}$, two cases occur:

- if $a<\frac{\theta_{1}}{2}$ : Notice first that one has $\theta_{1}-\frac{k_{3}}{2-\alpha}\left|y_{2}\right|^{2-\alpha}<$ $\frac{\theta_{1}}{2}$ which ensures that $\left|y_{2}\right|>\left(\frac{2-\alpha}{k_{3}} \frac{\theta_{1}}{2}\right)^{\frac{1}{2-\alpha}}$. Then the following inequalities

$$
\begin{aligned}
\dot{V} & \leq K_{1}\left(\frac{\theta_{1}}{2}\right)^{\frac{\alpha}{2-\alpha}} k_{3}\left|y_{2}\right|^{1-\alpha} \bar{p}-\gamma K_{1}\left|y_{2}\right|^{1+\alpha}+\gamma\left|y_{2}\right| \bar{p} \\
& \leq-\gamma K_{1}\left|y_{2}\right|^{1+\alpha}+\left[K_{1}\left(\frac{\theta_{1}}{2}\right)^{\frac{\alpha}{2-\alpha}} k_{3}+\gamma\right] \bar{p} \\
& \leq-\gamma K_{1}\left(\frac{2-\alpha}{k_{3}} \frac{\theta_{1}}{2}\right)^{\frac{1+\alpha}{2-\alpha}}+\left[K_{1} k_{3}+\gamma\right] \bar{p}
\end{aligned}
$$

are verified whenever

$$
\theta_{1}>\frac{2 k_{3}}{2-\alpha}\left[\frac{\left(K_{1} k_{3}+\gamma\right) \bar{p}}{\gamma K_{1}}\right]^{\frac{2-\alpha}{1+\alpha}}
$$

which ensures that $\dot{V}<0$.

- if $a>\frac{\theta_{1}}{2}$ : then one has

$$
\begin{aligned}
\dot{V} & \leq-K_{1}\left(\frac{\theta_{1}}{2}\right)^{\frac{\alpha}{2-\alpha}}\left[k_{3}\left|y_{2}\right|^{1-\alpha}\left\{K_{2}\left(\frac{\theta_{1}}{2}\right)^{\frac{\alpha}{2-\alpha}}-\bar{p}\right\}\right] \\
& -\gamma\left|y_{2}\right|\left[K_{1}\left|y_{2}\right|^{\alpha}-\bar{p}\right] . \\
& =-\left|y_{2}\right|^{1-\alpha}\left(\gamma K_{1}\left(\left|y_{2}\right|^{\alpha}\right)^{2}-\gamma \bar{p}\left|y_{2}\right|^{\alpha}\right. \\
& \left.+K_{1}\left(\frac{\theta_{1}}{2}\right)^{\frac{\alpha}{2-\alpha}} k_{3}\left\{K_{2}\left(\frac{\theta_{1}}{2}\right)^{\frac{\alpha}{2-\alpha}}-\bar{p}\right\}\right)
\end{aligned}
$$

which corresponds to a second order polynomial in $\left|y_{2}\right|^{\alpha}$. The polynomial has definite sign (positive) if its parameters satisfies the conditions:

1) $K_{2}\left(\frac{\theta_{1}}{2}\right)^{\frac{\alpha}{2-\alpha}}>2 \bar{p}$ and

2) $(\gamma \bar{p})^{2}<4 \gamma K_{1}^{2} k_{3}\left(\frac{\theta_{1}}{2}\right)^{\frac{\alpha}{2-\alpha}}\left\{K_{2}\left(\frac{\theta_{1}}{2}\right)^{\frac{\alpha}{2-\alpha}}-\bar{p}\right\}$.

These conditions lead to the following ones appearing in the proposition statement:

1) $\theta_{1}>2\left(\frac{2 \bar{p}}{K_{2}}\right)^{\frac{2-\alpha}{\alpha}}$ and 
2) $\theta_{1}>2\left[\frac{\left(1-K_{1} k_{3}\right) \bar{p}}{4 K_{1} K_{2} k_{3}}\right]^{\frac{2-\alpha}{\alpha}}$

In addition, $\dot{V}$ can be zero if $y_{2}=0$. This is not possible whenever $\left|y_{1}\right|>\left(\frac{\bar{p}}{K_{2}}\right)^{\frac{2-\alpha}{\alpha}}$ according to (9).

- Pick a positive constant $\theta_{2}$ and assume that $\left|y_{2}\right|^{2-\alpha}>$ $\frac{2-\alpha}{k_{3}} \theta_{2}$, two cases occur:

- if $a<\frac{\theta_{2}}{2}$ : Notice first that since $\theta_{2}-\left|y_{1}\right|<\frac{\theta_{2}}{2}$ then $\left|y_{1}\right|>\frac{\theta_{2}}{2}$. The following inequalities

$$
\begin{aligned}
\dot{V} & \leq K_{1} \frac{\theta_{2}}{2} k_{3}\left|y_{2}\right|^{1-\alpha} \bar{p}-\gamma K_{1}\left|y_{2}\right|^{1+\alpha}+\gamma\left|y_{2}\right| \bar{p} \\
& \leq-\gamma K_{1}\left|y_{2}\right|^{1+\alpha}+\left[K_{1} \frac{\theta_{2}}{2} k_{3}+\gamma\right] \bar{p} \\
& \leq-\gamma K_{1}\left(\frac{2-\alpha}{k_{3}} \theta_{2}\right)^{\frac{1+\alpha}{2-\alpha}}+\left[K_{1} k_{3}+\gamma\right] \bar{p}
\end{aligned}
$$

are verified whenever

$$
\theta_{2}>\frac{k_{3}}{2-\alpha}\left[\frac{\left(K_{1} k_{3}+\gamma\right) \bar{p}}{\gamma K_{1}}\right]^{\frac{2-\alpha}{1+\alpha}}
$$

- if $a>\frac{\theta_{2}}{2}:$ Then one has

$$
\begin{aligned}
\dot{V} & \leq-K_{1}\left(\frac{\theta_{2}}{2}\right)^{\frac{\alpha}{2-\alpha}}\left[k_{3}\left|y_{2}\right|^{1-\alpha}\left\{K_{2}\left(\frac{\theta_{2}}{2}\right)^{\frac{\alpha}{2-\alpha}}-\bar{p}\right\}\right] \\
& -\gamma K_{1}\left|y_{2}\right|^{\alpha+1}+\gamma \bar{p}\left|y_{2}\right| \\
& =-\left|y_{2}\right|^{1-\alpha}\left(\gamma K_{1}\left(\left|y_{2}\right|^{\alpha}\right)^{2}-\gamma \bar{p}\left|y_{2}\right|^{\alpha}\right. \\
& \left.+K_{1}\left(\frac{\theta_{2}}{2}\right)^{\frac{\alpha}{2-\alpha}} k_{3}\left\{K_{2}\left(\frac{\theta_{2}}{2}\right)^{\frac{\alpha}{2-\alpha}}-\bar{p}\right\}\right)
\end{aligned}
$$

which corresponds to a second order polynomial in $\left|y_{2}\right|^{\alpha}$. The polynomial has definite sign (positive) if its parameters satisfies the conditions:

1) $K_{2}\left(\frac{\theta_{2}}{2}\right)^{\frac{\alpha}{2-\alpha}}>2 \bar{p}$ and

2) $(\gamma \bar{p})^{2}<4 \gamma K_{1}^{2} k_{3}\left(\frac{\theta_{2}}{2}\right)^{\frac{\alpha}{2-\alpha}}\left\{K_{2}\left(\frac{\theta_{2}}{2}\right)^{\frac{\alpha}{2-\alpha}}-\bar{p}\right\}$.

These conditions lead to the following ones appearing in the proposition statement:

1) $\theta_{2}>2\left(\frac{2 \bar{p}}{K_{2}}\right)^{\frac{2-\alpha}{\alpha}}$ and

According to proposition 4.2, the trajectories of (5) converges within the ball

$$
\mathbb{B}_{1}=\left\{\left(z_{1}, z_{2}\right),\left|z_{1}\right| \leq \theta_{1},\left|z_{2}\right| \leq \theta_{2}+h p\right\} .
$$

\section{NUMERICAL ISSUES}

\section{A. Derivative estimation}

The system is equipped with a position sensor. However, its velocity is also needed for the controller implementation. A derivative estimator is given by:

$$
\tilde{x}_{2}(t)=\frac{6}{T^{3}} \int_{0}^{T}(T-2 s) x_{1}(t-s) d s .
$$

This estimator, which has been used in [9], is first introduced in [6]. The convolution (11) is numerically approximated by a discrete one. With $d$ to denote the sampling time, (11) is approximated by $\tilde{\tilde{x}}_{2}((k+1) d)=\sum_{i=0}^{7} h_{1}(i) \times x_{1}((k-i) d)$, $k \in \mathbb{N}$ where $h_{1}$ is a finite impulse response linear filter. It is given by $h_{1}=[13.89,37.04,9.26,0,-9.26,-37.04$, 13.89] for our application with $d=0.004$ and $T=d \times 7=$ 0.028 second.

\section{B. Numerical approximation of $z_{1}, z_{2}$ and $u$}

We assume that the delay $h$ is a multiple of the sampling time $d$, that is $h=n d$ and $n \in \mathbb{N} . \tilde{z}_{1}, \tilde{z}_{2}$ and $\tilde{u}$ are given by the following discrete convolutions:

$$
\begin{aligned}
\tilde{z}_{1}((k+1) d) & =x_{1}(k d)+h \tilde{\tilde{x}}_{2}(k d) \\
& +M\left(\frac{h u(k d-h)}{2}+\sum_{j=2}^{n-1}(j d) u((k-j) d)\right) \\
\tilde{z}_{2}((k+1) d) & =\tilde{\tilde{x}}_{2}(k d) \\
& +M\left(\frac{u(k d-h)}{2}+\sum_{j=2}^{n-1} u((k-j) d)+\frac{u(k d)}{2}\right) \\
\tilde{u}((k+1) d) & =-k_{1}\left\lfloor\tilde{z}_{2}(k d)\right\rceil^{\alpha} \\
& -k_{2}\left\lfloor\tilde{z}_{1}(k d)+\frac{k_{3}}{2-\alpha}\left\lfloor\tilde{z}_{2}(k d)\right\rceil^{2-\alpha}\right\rceil^{\frac{\alpha}{2-\alpha}}
\end{aligned}
$$

\section{EXPERIMENTATION}

\section{A. Platform description}

As depicted in figure 3, the platform is composed by the following elements: a servovalve (Festo, MPYE-5-1-1/8-LF010-B), a compressor and a pneumatic cylinder Festo, DNCI32-200-P-A-MU). This cylinder can be briefly described as a double effect cylinder with a piston diameter of $32 \mathrm{~mm}$ and a stroke length of $200 \mathrm{~mm}$. It is equipped with a built-in piston position sensor. The control law which is implemented in Matlab/Simulink is performed through a dSPACE 1103 microcontroller. The system monitoring in real time is realized by the ControlDesk software. Several experiments have been conducted in order to test the performances of the control law and to find the best value for the delay. In the sequel, all the parameters are fixed except the delay $h$. Note that tests are carried out with and without load for each delay value. The following tuning parameters are used $k_{1}=14, k_{2}=1$ and $k_{3}=0.1$.

\section{B. Experimental tests}

1) Test 1, h=0 second: The objective of this test is to demonstrate that a second order delay-free model is not representative of the pneumatic actuator. With $h=0$, one has $x=z=y$. Through this test with no load added to the cylinder, we can deduce that the value $h=0$ is not the convenient one, because figure 4 shows oscillations caused by the miss-modelling of the delay. 


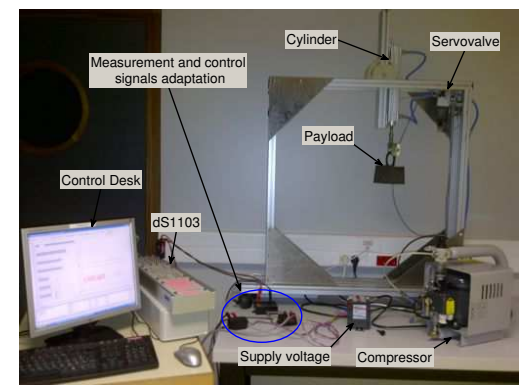

Fig. 3. Experimental setup

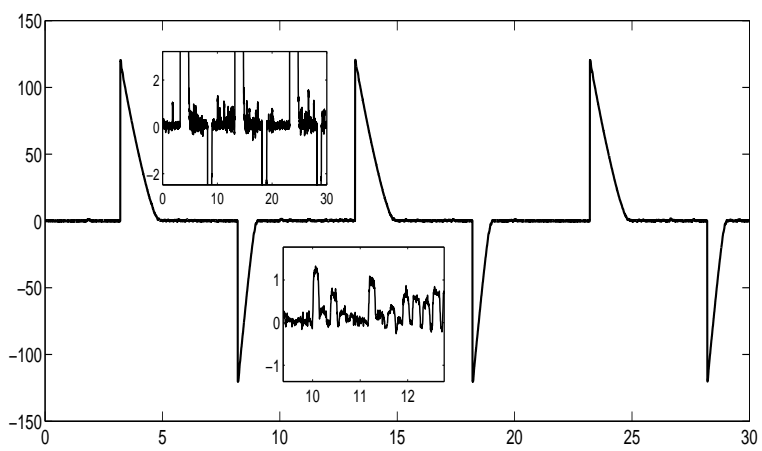

Fig. 4. Error versus time. $h=0, \alpha=0.1$, no load.

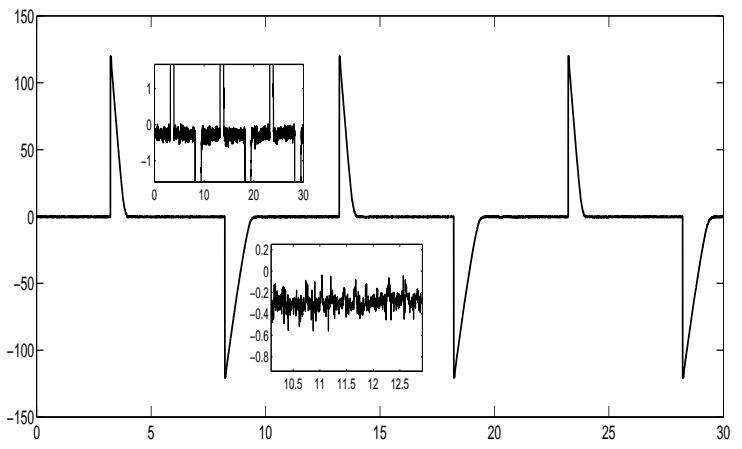

Fig. 5. Error versus time. $h=0.02, \alpha=0.1$, no load.

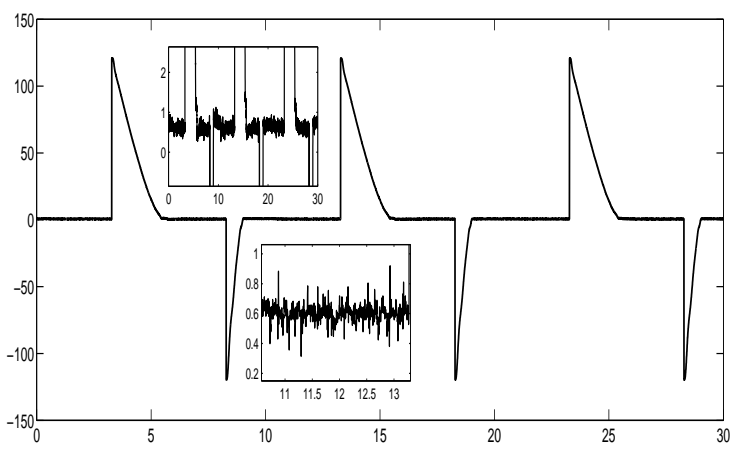

Fig. 6. Error versus time. $h=0.02, \alpha=0.1$, with added load.

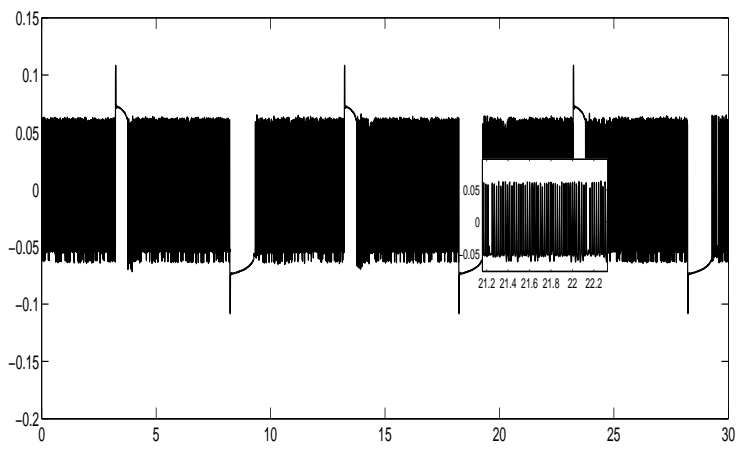

Fig. 7. Control effort versus time . $h=0.02, \alpha=0.1$, no load.

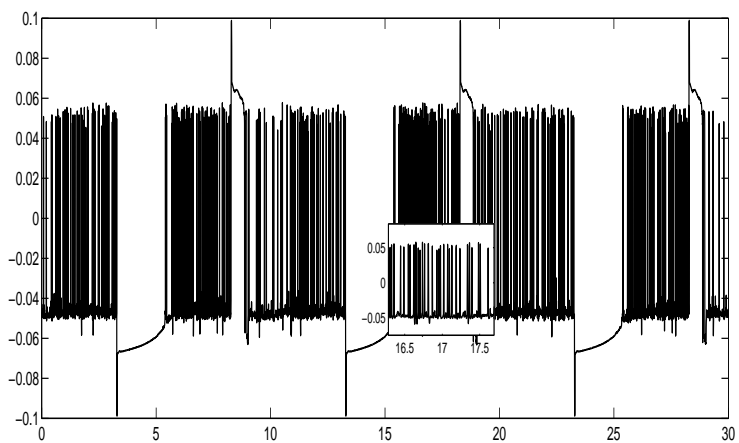

Fig. 8. Control effort versus time. $h=0.02, \alpha=0.1$, with added load.

2) Test 2, $h=0.02$ second: Better results are observed with a delay value $h=0.02$. The robustness of the control law is clearly proved in this case as depicted in figure 5 and 6 . Indeed, the static error is less than $0.4 \mathrm{~mm}$ without load and it is about $0.6 \mathrm{~mm}$ with an added load. Figure 7 and 8 correspond to the input control signal where a chattering (see equation 6) with frequency approximately about $50 \mathrm{~Hz}$ can be perceived. It is an acceptable frequency since the natural frequency of oscillation of the servovalve is $100 \mathrm{~Hz}$.

3) Test 3, $h=0.06$ second: A delay of 0.06 second is not adequate since the experiments revealed a much bigger static error (see figures 9, 10, 11 and 12).

\section{CONCLUSION}

A second order model with delayed input is proposed to represent the dynamics of the pneumatic actuator. A homogeneous robust nonlinear controller is synthesized based on the proposed model. The validity of the model as well as the controller were demonstrated experimentally.

\section{REFERENCES}

[1] Artstein Z., "Linear systems with delayed controls: a reduction". IEEE Transactions on automatic control, Vol. 27, No. 4, pp. 869-879, 1982.

[2] Bhat P. and Bernstein D.S., "Continous Finite-Time Stabilization of the Translational and Rotational Double Integrators", IEEE Transactions on automatic control, Vol. 27, No. 4, MAY 1998.

[3] Bhat P. and Bernstein D.S., "Finite time stability of homogeneous systems", Proceedings of the ACC, New Mexico, 1997. 


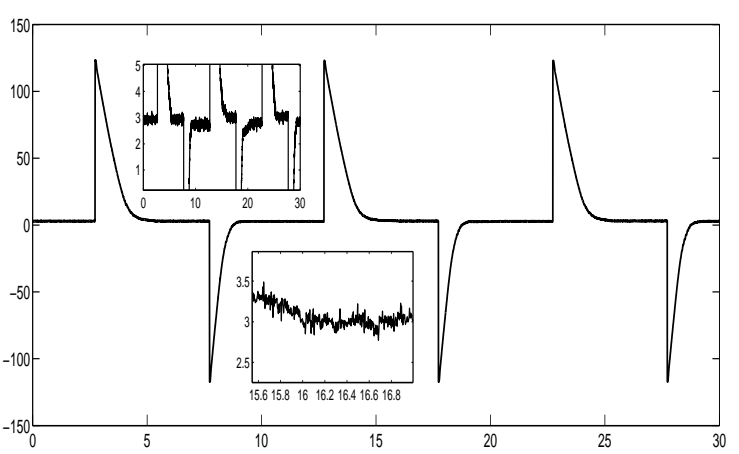

Fig. 9. Error effort versus time. $h=0.06, \alpha=0.1$, no load.

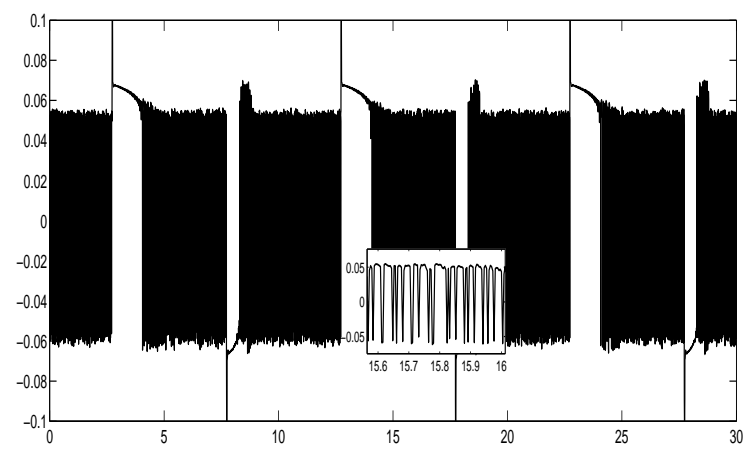

Fig. 10. Control effort versus time. $h=0.06, \alpha=0.1$, no load.

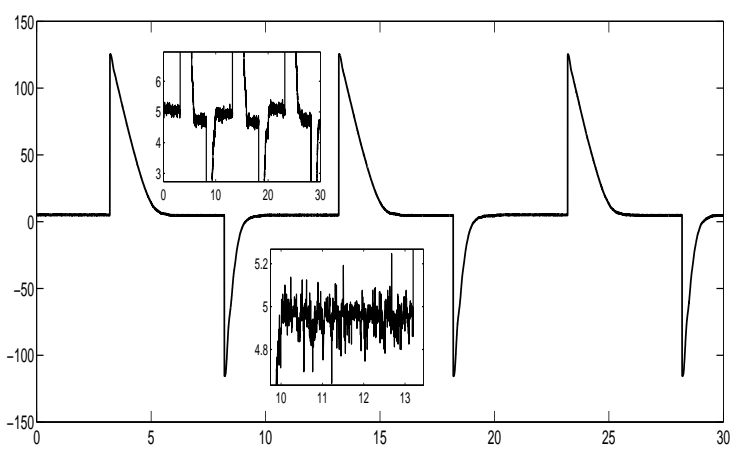

Fig. 11. Error effort versus time. $h=0.06, \alpha=0.1$, with added load.

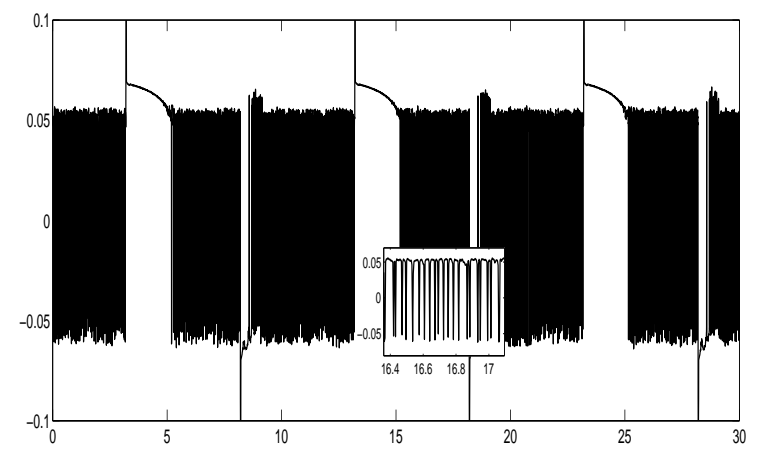

Fig. 12. Control effort versus time. $h=0.06, \alpha=0.1$, with added load.
[4] Bone G.M. and Ning S., "Experimental Comparison of Position Tracking Control Algorithms for Pneumatic Cylinder Actuators". IEEE/ASME Transactions on mechatronics, Vol. 12, No. 5, pp. 557561, 2007.

[5] Kokotovic P., Khalil H.K. and O'Reilly J., "Singular Perturbation Methods in Control: Analysis and Design". SIAM, Classics In Applied Mathematics, 1999

[6] Mboup M., Join C. and Fliess M., "Numerical differentiation with annihilators in noisy environment". Numerical Algorithms Vol. 50, No. 4 pp. 439-467, 2009.

[7] Nguyen T., Leavitt J., Jabbari F. and Borbow J.E., "Accurate slidingmode control of pneumatic systems using low cost solenoid valves". Transactions on mechatronics Vol. 12, No. 2 pp 216-219, 2007.

[8] Rao Z. and Bone G.M., "Nonlinear Modeling and Control of Servo Pneumatic Actuators". Trans. on control systems technology Vol. 16, No. 3, pp. 562-569, 2008.

[9] Riachy S. and Ghanes M. "A nonlinear controller for pneumatic servo systems: Design and experimental tests". Accepted in IEEE/ASME Transactions on mechatronics, 2013. DOI 10.1109/TMECH.2013.2280956

[10] Wang J., Pu J. and Moore P., "A practical control strategy for servopneumatic actuator systems". Control Engineering Practice No.7 pp. 1483-1488, 1999.

[11] Tressler J.-M., Clement T., Kazerooni H. and Lim M. "Dynamic behavior of pneumatic systems for lower extremity extenders.". Proceedings of IEEE international conference on robotics and automation, pp. 3248-3253, 2002. 